\title{
Synthesis of Interdigital Capacitors Based on Particle Swarm Optimization and Artificial Neural Networks
}

\author{
Jehad Ababneh, Majid Khodier, Nihad Dib \\ Electrical Engineering Department, Jordan University of Science and Technology, Irbid, Jordan \\ Received 28 February 2005; accepted 1 June 2005
}

\begin{abstract}
This article reports on the use of the particle swarm optimization (PSO) algorithm in the synthesis of the planar interdigital capacitor (IDC). The PSO algorithm is used to optimize the geometry parameters of the IDC in order to obtain a certain capacitance value. The capacitance value of the IDC is evaluated using an artificial neural network (ANN) model with the geometry parameters of the IDC as its inputs. Several design examples are presented that illustrate the use of the PSO algorithm, and the design goal in each example is easily achieved. Full-wave electromagnetic simulations are also performed for some of the studied IDC structures implemented using coplanar waveguide (CPW) technology. The simulation results are in good agreement with those obtained using the ANN/PSO algorithm. (C) 2006 Wiley Periodicals, Inc. Int J RF and Microwave CAE 16: 000-000, 2006.
\end{abstract}

Keywords: neural networks; particle swarm optimization; coplanar waveguide; interdigital capacitor

\section{INTRODUCTION}

One of the common elements in microwave integrated circuits (MICs) is the interdigital capacitor (IDC), which was first introduced and analyzed by Alley [1] almost 35 years ago. Since then, it has been the subject of several publications [2-12]. Given the recent developments in wireless telecommunications, a fast, accurate, and effective computer-aided design (CAD) model to design IDCs has become increasingly more important in microwave design [13].

In a recent work [14], we developed an artificial neural network (ANN) model to compute the capacitance of the IDC shown in Figure 1. A closed-form expression for the capacitance was used to generate data for training and testing. The expression was

Correspondence to: J. Ababneh; email: ababnehj@just.edu.jo. DOI 10.1002/mmce.20141

Published online in Wiley InterScience (www.interscience.wiley. com).

(C) 2006 Wiley Periodicals, Inc. taken from [8] with few modifications. Using that expression, a CAD model for the coplanar waveguide (CPW) IDC was presented in [14].

The objective of this article is to use the developed ANN model in [14], coupled with the particle swarm optimization (PSO) algorithm [15], to synthesize the IDC shown in Figure 1. That is, given a specific capacitance value, the dimensions of the IDC and the substrate parameters will be obtained. Alternatively, one can fix the substrate parameters and find the corresponding IDC physical dimensions and number of fingers. It should be noted that no ground plane is shown in Figure 1. However, our design works for the series capacitance of the CPW IDCs [14], while the shunt capacitors can be derived using expressions given in [14]. Moreover, our design should work for microstrip IDCs with a ground plane far enough such that it has negligible effect on the series capacitance of the IDC [14].

A brief description of the developed ANN model used in [14] is presented in the next section, while the 


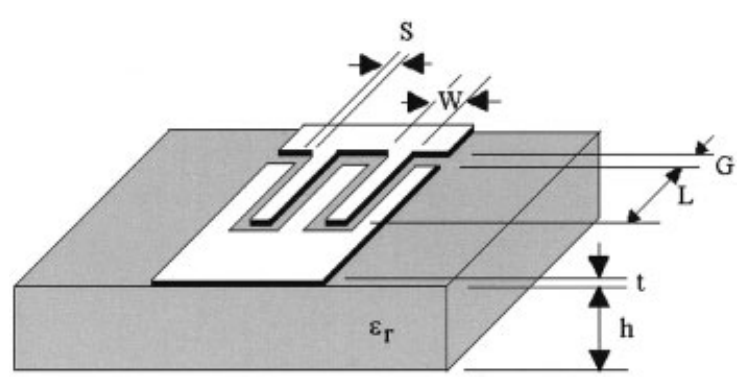

Figure 1. General view of a five-finger interdigital capacitor.

PSO algorithm is described in section III. Several design cases are given in section IV. To support the theory presented in this article, full-wave electromagnetic simulations realized using the commercial software IE3D are also presented for some of the IDC structures, and agreement with the ANN/PSO results is good.

\section{ANN MODEL}

Recently, ANNs have been widely used in RF and microwave CAD [16-22]. The multilayer feed-forward network is one of the most effective and commonly used ANN architectures [23]. This type of network consists of neurons that are arranged in layers (an input layer, output layer, and a number of hidden layers), as shown in Figure 2.

In [14], a multilayer feed-forward network was developed to model the capacitance of the IDC. The parameters: $\varepsilon_{r}, h, W, S, t$, and $G$ of the IDC are the inputs to the network, while the network outputs three variables from which the value of the IDC capacitance, for some given values of $N$ and $L$, is obtained directly using a simple expression given in [14]. Table I gives the range of the input parameters used in

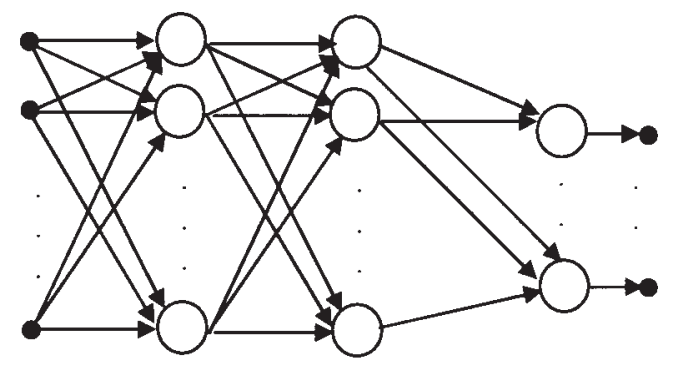

Input Layer

Hidden Layers

Output Layer

Figure 2. Example of a multilayer feed-forward network architecture with an input layer, two hidden layers, and an output layer.
TABLE I. The Range of Input Parameters

\begin{tabular}{lccccc}
\hline$\varepsilon_{r}$ & $h[\mu \mathrm{m}]$ & $W[\mu \mathrm{m}]$ & $S[\mu \mathrm{m}]$ & $t[\mu \mathrm{m}]$ & $G[\mu \mathrm{m}]$ \\
\hline $9-13$ & $100-700$ & $10-50$ & $2-50$ & $1-5$ & $2-50$ \\
\hline
\end{tabular}

creating the training and testing data. This range covers most practical CPW IDCs used in MMICs.

The network is trained and tested using 1000 and 100 different randomly distributed points of the parameters in the range given in Table I, respectively. A two-hidden layer network with 15 neurons in the first layer and 25 neurons in the second layer successfully models the IDC. The developed ANN can be used to accurately compute the value of the capacitance of the IDC for any given set of input parameters in negligible time. The ANN results given in [14] demonstrate the accuracy and the generalization ability of the trained ANN model. Therefore, the developed network is an accurate and attractive alternative module that can be used in a CAD-based tool to optimize the design of an IDC. The following sections support and demonstrate this conclusion.

\section{PARTICLE SWARM OPTIMIZATION}

The PSO algorithm is used in this article to optimize the geometry parameters of the IDC in order to obtain a certain capacitance value. The PSO algorithm is an evolutionary algorithm capable of solving general multidimensional optimization problems. The PSO algorithm was introduced in 1995 by Kennedy and Eberhart [24], and since then the PSO has gained an increasing popularity as an efficient alternative to genetic algorithms (GAs) and simulated annealing (SA) in solving optimization design problems. Like other evolutionary algorithms, the PSO algorithm utilizes the social interaction between independent agents, hereafter called particles, during their search for the optimum solution using the concept of fitness. What is attractive about the PSO algorithm over conventional and evolutionary optimization algorithms is its simplicity and generality. It requires the least amount of mathematical preprocessing. It is easy to write a general-purpose computer code for the PSO and in it the fitness evaluation is left for the user to conduct, depending on the problem at hand. The PSO algorithm has been tested on standard optimization problems ranging in difficulty from simple zero-finding of $\mathrm{N}$-dimensional functions to the optimization of the profile of corrugated horn antennas with predefined radiation properties [15]. 


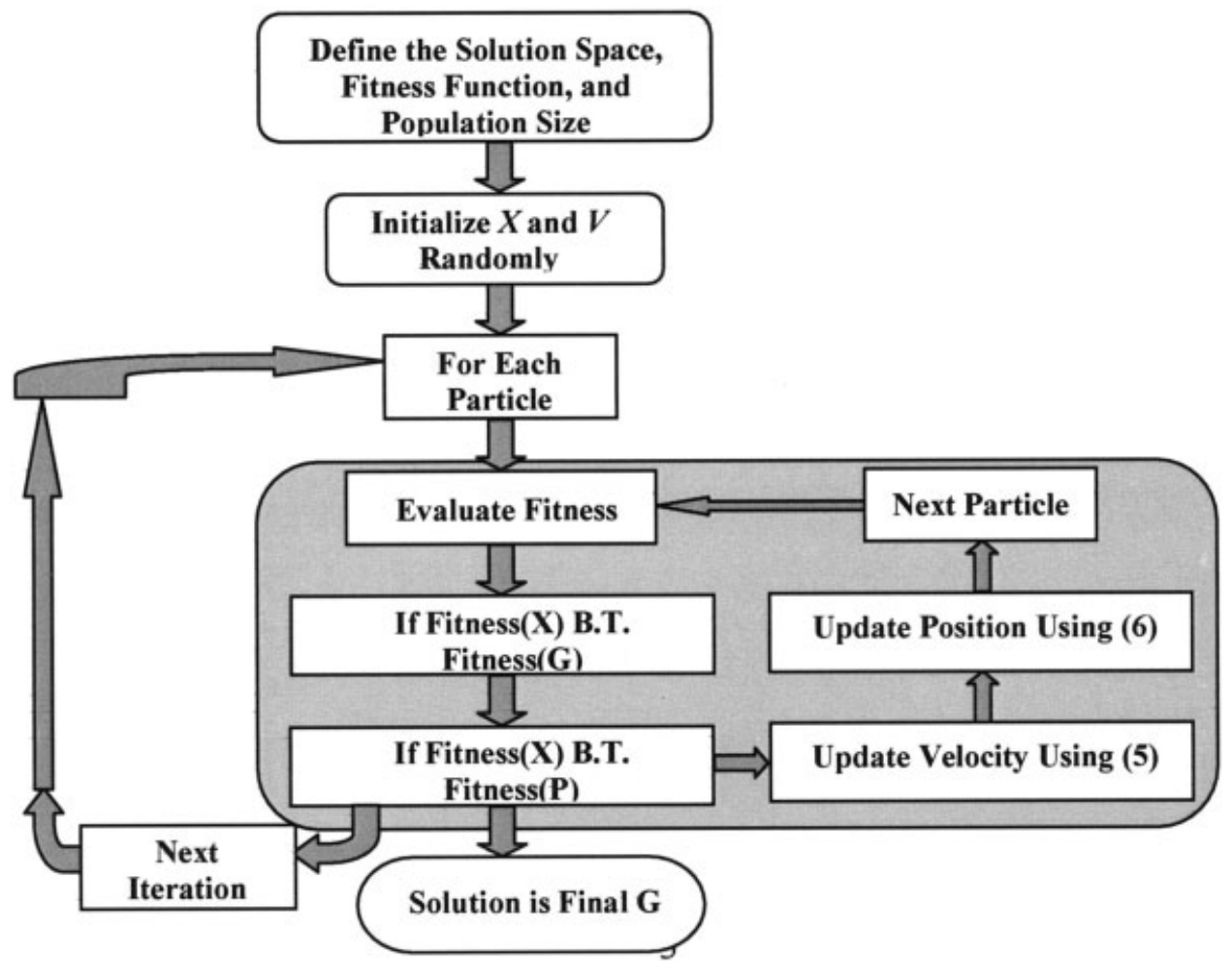

Figure 3. Flowchart diagram showing the main steps of the PSO algorithm. B.T. stands for "better than" and for the current problem it is translated mathematically as "less than."

It should be mentioned that this article is not intended to be an extensive review of the PSO algorithm, and therefore only the main steps of the algorithm are presented here. The reader is referred to $[15$, 24-30] and the references mentioned therein for a detailed discussion of the basic concepts of the PSO algorithm, how it works, and its advantages over other optimization techniques. The flowchart diagram shown in Figure 3 summarizes the main steps of the PSO algorithm discussed below.

After defining the solution space and the fitness function, the PSO algorithm starts by randomly initializing the position and velocity of each particle in the swarm. For an $N$-dimensional problem with $M$ particles, each particle has its own position and velocity in every dimension; therefore, the positions and velocities of the particles can be specified by the following $M \times N$ matrices:

$$
X=\left[\begin{array}{cccc}
x_{11} & x_{12} & \ldots & x_{1 N} \\
x_{21} & x_{22} & \ldots & x_{2 N} \\
& & \ddots & \\
x_{M 1} & x_{M 2} & \ldots & x_{M N}
\end{array}\right],
$$

$$
V=\left[\begin{array}{cccc}
v_{11} & v_{12} & \ldots & v_{1 N} \\
v_{21} & v_{22} & \ldots & v_{2 N} \\
& & \ddots & \\
x_{M 1} & v_{M 2} & \ldots & v_{M N}
\end{array}\right] .
$$

Any row of the position matrix represents a possible solution to the optimization problem. The velocity of each particle depends on the distance between the current position and the positions that result in good fitness values. To update the velocity matrix, every particle should know, up to the current iteration, its personal-best and the global-best position vectors. The personal-best position vector defines the position at which each particle attained its best fitness value up to the present iteration. The personal best position can be defined by the following matrix:

$$
P=\left[\begin{array}{cccc}
p_{11} & p_{12} & \ldots & p_{1 N} \\
p_{21} & p_{22} & \ldots & p_{2 N} \\
& & \ddots & \\
p_{M 1} & p_{M 2} & \ldots & p_{M N}
\end{array}\right]
$$


TABLE II. IDC Design Results (Eight Parameters)

\begin{tabular}{cccccccccc}
\hline$C_{\text {des }}[\mathrm{pF}]$ & $\varepsilon_{r}$ & $h[\mu \mathrm{m}]$ & $W[\mu \mathrm{m}]$ & $S[\mu \mathrm{m}]$ & $t[\mu \mathrm{m}]$ & $G[\mu \mathrm{m}]$ & $L[\mu \mathrm{m}]$ & $N$ & $C_{A N N}[\mathrm{pF}]$ \\
\hline 0.05 & 9 & 303 & 27 & 30 & 1 & 27 & 292 & 4 & 0.0499 \\
& 11 & 300 & 35 & 32 & 1 & 34 & 301 & 3 & 0.0498 \\
0.1 & 9 & 496 & 17 & 22 & 1 & 17 & 250 & 10 & 0.099 \\
0.5 & 12.61 & 505 & 35 & 27 & 3 & 28 & 254 & 29 & 0.498 \\
1 & 12.88 & 301 & 19 & 12 & 5 & 18 & 301 & 50 & 1.003 \\
1.5 & 11 & 302 & 17 & 13 & 4 & 16 & 602 & 49 & 1.518 \\
& 12 & 301 & 21 & 17 & 2 & 17 & 601 & 46 & 1.490 \\
\hline
\end{tabular}

The row of the position matrix $X$ at which the best fitness value is achieved is the global-best position vector in the solution space, given by

$$
G=\left[\begin{array}{llll}
g_{1} & g_{2} & \ldots & g_{N}
\end{array}\right]
$$

All the information needed by the PSO algorithm is contained in $X, V, P$, and $G$. The core of the PSO algorithm is how to update these matrices in each iteration of the algorithm. These matrices are updated such that the position vector of each particle is encouraged to move closer to both its personal-best and the global-best positions at every new iteration. For this goal, the velocity matrix is updated according to $[25,26]$ :

$v_{m n}^{t}=w v_{m n}^{t-1}+c_{1} \eta_{n 1}^{t}\left(p_{m n}^{t}-x_{m n}^{t-1}\right)+c_{2} \eta_{n 2}^{t}\left(g_{n}^{t}-x_{m n}^{t-1}\right)$,

where the superscripts $t$ and $t-1$ refer to the time index of the current and the previous iterations, $\eta_{n 1}$ and $\eta_{n 2}$ are two different uniformly-distributed random numbers in the interval $[0,1]$. The parameters $c_{1}$ and $c_{2}$ specify the relative weight of the personal-best position versus the global-best position. It has become a common practice to choose a value of 2.0 for both parameters [29]. The parameter $w$ is called the "inertial weight", and it is a number in the range $[0,1]$ that specifies the weight by which the particle's current velocity depends on its previous velocity, and the distance between the particle's position and its personal-best and global-best positions. Empirical studies [15] have shown that the PSO algorithm converges faster if $w$ is linearly damped with iterations, for example, starting at 0.9 at the first iteration and finishing at 0.4 in the last iteration. The position matrix is updated according to the simple relation:

$$
X^{t}=X^{t-1}+V^{t}
$$

The PSO algorithm, like GA and other evolutionary algorithms, uses the concept of fitness to decide how good the position vector of each particle in the swarm is, during the search for the optimum position vector in the $\mathrm{N}$-dimensional space. At each iteration of the algorithm, the particle's position vector that resulted in the best fitness is chosen as the global-best position vector, and this information is communicated to all other particles to use in updating the velocity and position vectors matrices according to eqs. (5) and (6). In the current problem, we are interested in designing the geometry of the IDC to give us a certain capacitance value. To achieve this goal, the following function is used to evaluate the fitness:

$$
\text { Fitness }=\min \left|C_{d e s}-C_{A N N}\right|,
$$

where $C_{d e s}$ is the desired capacitance, and $C_{A N N}$ is the capacitance evaluated form the ANN model as outlined in section II. It is clear from (7) that for the current problem, the particle with the best fitness is the one with the position vector that minimizes the fitness function defined in (7).

\section{RESULTS AND DISCUSSION}

The PSO algorithm and the ANN model were used to design the IDC. In designing the IDC, we aim to find

TABLE III. IDC Design Results (Seven Parameters, $\varepsilon_{r}=13$ )

\begin{tabular}{ccccccccc}
\hline$C_{\text {des }}[\mathrm{pF}]$ & $h[\mu \mathrm{m}]$ & $W[\mu \mathrm{m}]$ & $S[\mu \mathrm{m}]$ & $t[\mu \mathrm{m}]$ & $G[\mu \mathrm{m}]$ & $L[\mu \mathrm{m}]$ & $N$ & $C_{\text {ANN }}[\mathrm{pF}]$ \\
\hline \multirow{2}{*}{0.5} & 310 & 25 & 30 & 4 & 25 & 306 & 27 & 0.494 \\
& 306 & 32 & 25 & 4 & 25 & 312 & 24 & 0.497 \\
1.5 & 337 & 20 & 19 & 3 & 22 & 601 & 44 & 1.492 \\
& 302 & 20 & 21 & 3 & 19 & 601 & 45 & 1.491 \\
\hline
\end{tabular}


TABLE IV. IDC Design Results (Seven Parameters, $\varepsilon_{r}=9.8$ )

\begin{tabular}{ccccccccc}
\hline$C_{\text {des }}[\mathrm{pF}]$ & $h[\mu \mathrm{m}]$ & $W[\mu \mathrm{m}]$ & $S[\mu \mathrm{m}]$ & $t[\mu \mathrm{m}]$ & $G[\mu \mathrm{m}]$ & $L[\mu \mathrm{m}]$ & $N$ & $C_{A N N}[\mathrm{pF}]$ \\
\hline \multirow{2}{*}{0.5} & 516 & 19 & 24 & 1 & 17 & 505 & 25 & 0.502 \\
& 318 & 29 & 13 & 4 & 28 & 509 & 18 & 0.496 \\
\multirow{2}{*}{1.5} & 411 & 34 & 10 & 4 & 14 & 607 & 42 & 1.504 \\
& 519 & 37 & 6 & 5 & 27 & 516 & 42 & 1.489 \\
\hline
\end{tabular}

its parameters that will result in a certain capacitance. In Table II, the dimensions of the IDC and the substrate parameters were determined for a given specific capacitance value. That is, starting with a desired value of capacitance $C_{d e s}$, the PSO algorithm is used to search the solution space for the dimensions of the IDC and the substrate parameters that will give the desired capacitance with some acceptable error. The designed values of the capacitance using the parameters obtained by the PSO search are either exactly equal or very close to the required values. The difference (if any) between the desired capacitance and the designed capacitance is a result of two factors: (i) the stopping criteria used in the PSO search and (ii) the allowance of only integer parameter values (except for the value of $\varepsilon_{r}$ ) to be a valid solution. In addition, Table II shows different sets of the dimensions of the IDC and the substrate parameters that give a certain required capacitance value. For example, two sets of parameters are shown for the desired capacitance values of 0.05 and $1.5 \mathrm{pF}$ which would give the designer more freedom in selecting the IDC parameters for a certain capacitance. Furthermore, it also emphasizes the fact that the parameters obtained from the PSO are not unique and depend on the random initialization step for this particular design problem.

In certain situations, some of the design parameters are fixed or given to the designer. For example, one can fix one or more of the IDC parameters and search for the other parameters. In Tables III and IV, the substrate type is fixed and the other parameters are obtained using the PSO algorithm. Table V shows the design results for fixed substrate parameters (the height and the type of the substrate) and metal thickness.

In all of the results shown in Tables II-V, the objective of the optimization is to find the IDC parameters that result in a capacitance value that is very close to the desired capacitance value. However, during the search process only values that are within the ranges given in Table I (which cover most practical IDCs) are used. Therefore, there are constraints on the values of the parameters that are accepted as a valid solution. In addition, more parameters were set to some desired values as we progress from Table II to Table V. For example, in Tables III and IV the dielectric constant of the substrate is set to some typical values, while in Table $\mathrm{V}$ the height, dielectric constant, and metal thickness are set to some fixed values which are assumed to be manufacturing standards. More constraints may be added on the other geometrical parameters, but this action may result in two problems. First, the solution space will be reduced, which in some cases might make the acceptable solution infeasible, especially for a high desired capacitance value. Second, in some other cases, this action could slow the search process dramatically.

In each row in Tables II-V, a nonunique solution that results in an acceptable error between the desired and the obtained capacitance values is given. The nonuniqueness of the solution was demonstrated by giving more than one solution for the same desired capacitance value, as shown in Tables III and IV. Three factors have led into this nonuniqueness of the solution. The fact that a desired capacitance value can be realized by many different parameters sets is the

TABLE V. IDC Design Results (Five Parameters, $\varepsilon_{r}=12.9, h=400 \mu \mathrm{m}, t=3 \mu \mathrm{m}$ )

\begin{tabular}{lcccccc}
\hline$C_{\text {des }}[\mathrm{pF}]$ & $W[\mu \mathrm{m}]$ & $S[\mu \mathrm{m}]$ & $G[\mu \mathrm{m}]$ & $L[\mu \mathrm{m}]$ & $N$ & $C_{A N N}[\mathrm{pF}]$ \\
\hline 0.155 & 46 & 20 & 30 & 534 & 4 & 0.154 \\
0.5 & 21 & 12 & 21 & 403 & 19 & 0.499 \\
0.75 & 34 & 35 & 18 & 319 & 36 & 0.750 \\
1 & 47 & 10 & 21 & 307 & 37 & 0.999 \\
1.5 & 48 & 6 & 48 & 306 & 50 & 1.499 \\
1.75 & 47 & 2 & 7 & 410 & 43 & 1.750 \\
2 & 44 & 2 & 4 & 50 & 2 \\
\hline
\end{tabular}



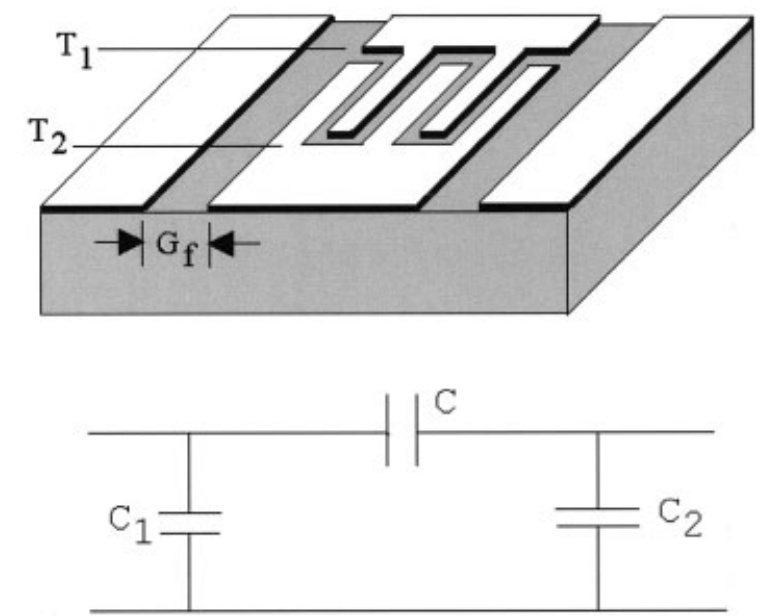

Figure 4. A general view of a CPW IDC and its equivalent-circuit model. $T_{1}$ and $T_{2}$ are the reference planes at which the scattering parameters are evaluated. $G_{f}$ was chosen to obtain $50 \Omega$ feeding lines.

most important one. The other two factors are the random initialization step and the velocity and position update of each particle according to eqs. (5) (which requires two random variables) and (6) in the PSO algorithm. One may argue that the PSO algorithm can be initialized with some desired initial parameters values to remove nonuniqueness of the solution. However, the other two factors are dominant and will again result in a nonunique solution.

During the synthesis process, the PSO algorithm will converge and the search process will be terminated if the difference between the desired capacitance value and the calculated (using the ANN model) capacitance value is less than or equal to an accepted error value. In this particular synthesis problem, an error value of $10^{-2}$ was used. Therefore, termination of the search process and the output of the PSO algorithm are controlled by the accepted error value. However, this does not mean that any set of parameters will be accepted as long as the termination criterion is achieved, but during the search process only the set of parameters in the solution space is used. As stated above, the solution space is the values of the parameters that are within the ranges given in Table I. It should be emphasized that these ranges cover most practical IDCs. As a result, the designer is not taking any risk by accepting the output of the PSO algorithm. In addition, the designer may obtain another solution simply by running the PSO algorithm one more time. Furthermore, the designer can set some of the parameters to some desired values and run the PSO algorithm to find the remaining parameters. Also, more constraints can be added on the parameters to change the solution space. However, careful attention to the selection of the parameters to be set or the constraints to be added should be made in order to avoid the possible occurrence of the two problems mentioned above.

To verify our approach, the full-wave electromagnetic simulator IE3D [31] is used to obtain the scattering parameters for some of the IDC designs presented in the above tables. To be able to perform a full-wave analysis, a ground plane needs to be added to the IDC structure shown in Figure 1. We have chosen to analyze a CPW IDC in which the ground planes are placed around the IDC, as shown in Figure 4. In the same figure, a lumped-element equivalent circuit of the CPW IDC is also shown [14]. The values of these capacitances are obtained from the scattering parameters using simple network theory [32]. Although, in this article, we are interested in the series capacitance $C$, values for the shunt capacitances $C_{1}$ and $C_{2}$ will be also presented and compared to those obtained using the expression proposed in [14]. It should be mentioned that if $N$ is even, the IDC structure will be symmetric and $C_{1}=C_{2}$, but if $N$ is odd, the structure is asymmetric and $C_{1} \neq C_{2}$.

The simulation results for some examples are shown in Figures 5-7. The results show that up to about $10 \mathrm{GHz}$ there is fair agreement between the value of the series capacitance from full-wave simulation and that obtained from the ANN model. The difference between the two values can be attributed to two reasons. First, the ANN model used is based on data generated from quasi-static expressions for the capacitance obtained using the conformal mapping method. Second, the circuit model used here neglects

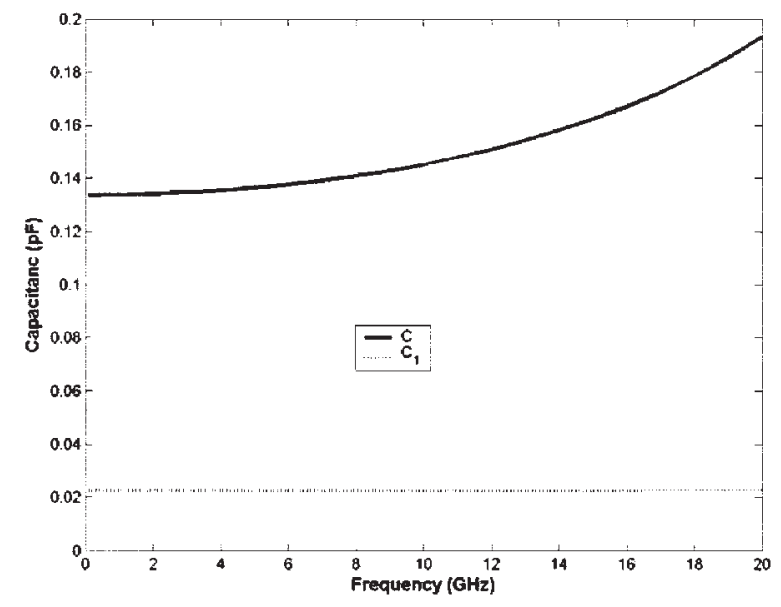

Figure 5. CPW IDC series and shunt capacitances vs. frequency. The corresponding geometry is given in the first row of Table II. The desired value of $C$ is $0.05 \mathrm{pF}$. $G_{f}=72$ $\mu \mathrm{m}$. 
the series inductance of the IDC, and that explains why the full-wave simulation results for the series capacitance are larger than the ANN model values in order to account for the neglected inductance.

Regarding the shunt capacitances, it can be seen that the value of this capacitance is almost independent of frequency. As explained in [14], the shunt capacitance can be approximated as the product of the IDC length with the per unit length capacitance of a CPW line having one ground plane only (with slot width $S_{f}$ and conductor width $W$ ). Such a line is a quasi-TEM line, which gives a constant shunt capacitance as a function of frequency. The values obtained using the expression in [14] are $C_{1}=C_{2}=0.021$, 0.0142 , and $0.046 \mathrm{pF}$ for the IDCs studied in Figures 5,6 , and 7 , respectively. These approximate values do not take into account the capacitance between the inner fingers and the ground plane. This explains why these values are less than those obtained from fullwave simulations (especially for the case with $N=$ 10, as shown in Fig. 6)

\section{CONCLUSION}

This article has illustrated the use of the particle swarm optimization (PSO) method in the synthesis of interdigital capacitors (IDCs). The PSO algorithm, in conjunction with an ANN model of the IDC, were successfully used to optimize the geometry parameters of the IDC in order to obtain a desired capacitance value. The validity of the presented design approach was shown through the discussion of several design examples. In each example, the design goal was easily

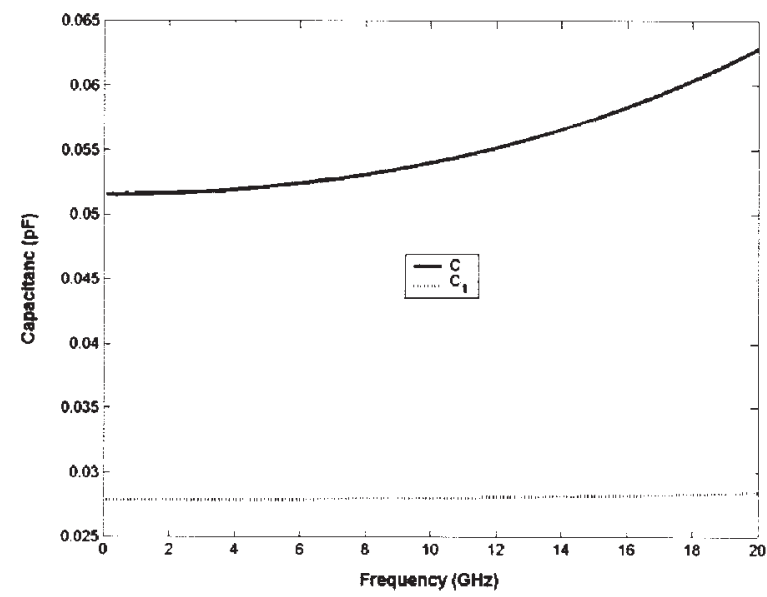

Figure 6. The CPW IDC series and shunt capacitances vs. frequency. The corresponding geometry is given in the third row of Table II. The desired value of $C$ is $0.1 \mathrm{pF}$. $G_{f}=130$ $\mu \mathrm{m}$.

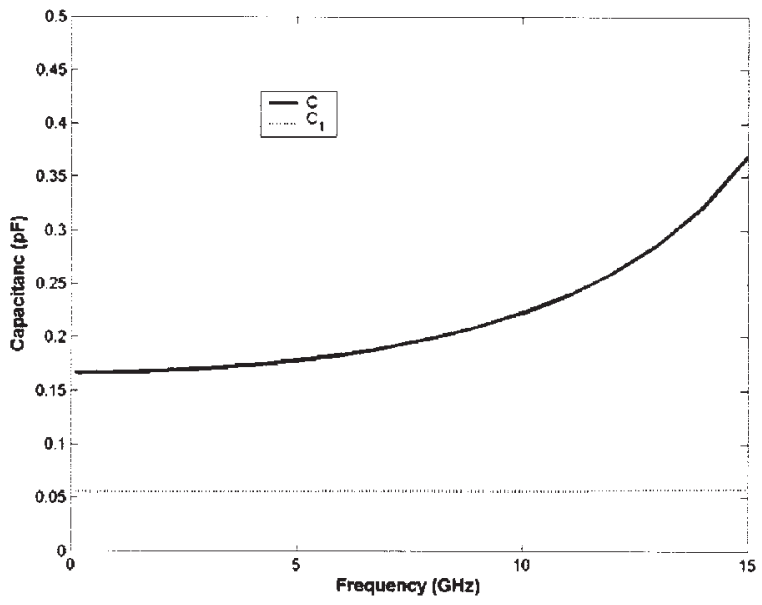

Figure 7. The CPW IDC series and shunt capacitances vs. frequency. The corresponding geometry is given in the first row of Table V. The desired value of $C$ is $0.155 \mathrm{pF}$. $G_{f}=$ $152 \mu \mathrm{m}$

achieved, at least in theory. The full-wave simulation results are also in support of the proposed ANN/PSO model of the IDC. A CAD model for the IDC based on the design methodology presented in this article can be easily incorporated with existing CAD software.

\section{REFERENCES}

1. G. Alley, Interdigital capacitors and their application to lumped-element microwave integrated circuits, IEEE Trans Microwave Theory Tech 18 (1970), 1028-1033.

2. J. Hobdell, Optimization of interdigital capacitors, IEEE Trans Microwave Theory Tech 27 (1979), 788 791.

3. R. Esfandiari, D. Maki, and M. Siracusa, Design of interdigital capacitors and their application to gallium arsenide monolithic filters, IEEE Trans Microwave Theory Tech 31 (1983), 57-64.

4. E. Pettenpaul, H. Kapusta, A. Weisgerber, H. Mampe, J. Luginsland, and I. Wolff, CAD models of lumped elements on GaAs up to $18 \mathrm{GHz}$, IEEE Trans Microwave Theory Tech 36 (1988), 294-304.

5. V. Sadhir, I. Bahl, and D. Willems, CAD-compatible accurate models of microwave passive elements for MMIC applications, Int J Microwave and MillimeterWave CAE 4 (1994), 148-162.

6. H. Wu, Z. Zhang, F. Barnes, C. Jackson, A. Kain, and J. Cuchiaro, Voltage tunable capacitors using hightemperature supercondcutors and ferroelectrics, IEEE Trans Appl Superconductivity 4 (1994), 156-160.

7. C. Chi and G. Rebeiz, Planar microwave and millimeter-wave lumped elements and coupled-line filters using micro-machining techniques, IEEE Trans Microwave Theory Tech 43 (1995), 730-738. 
8. S. Gevorgian, T. Martinsson, P. Linner, and E. Kolberg, CAD models for multilayered substrate interdigital capacitors, IEEE Trans Microwave Theory Tech 44 (1996), 896-904.

9. G. Coen, D. De Zutter, and N. Fache, Automatic derivation of equivalent circuits for general microstrip interconnection dicontinuities, IEEE Trans Microwave Theory Tech 44 (1996), 1010-1016.

10. L. Zhu and K. Wu, Accurate circuit model of interdigital capacitor and its application to design of new quasilumped miniaturized filters with suppression of harmonic resonance, IEEE Trans Microwave Theory Tech 48 (2000), 347-356.

11. D. Caratelli and R. Cicchetti, A full-wave analysis of interdigital capacitors for planar integrated circuits, IEEE Trans Magn 39 (2003), 1598-1601.

12. N. Dib, Q. Zhang, and U. Rohde, New CAD model of the microstrip interdigital capacitor, Active and Passive Electron Compon 27 (2004), 237-245.

13. R.-Y. Yang, M.-H. Weng, C.-Y. Hung, H.-J. Chen, and M.-P. Houng, Novel compact microstrip interdigital bandstop filters, IEEE Trans Ultrasonics Ferroelect and Freq Control 51 (2004), 1022-1025.

14. N. Dib, J. Ababneh, and A. Omar, CAD modeling of coplanar waveguide interdigital capacitor, Int J RF and Microwave CAE 15 (2005), 551-559.

15. J. Robinson and Y. Rahmat-Sami, Particle swarm optimization in electromagnetics, IEEE Trans Antennas Propagat 52 (2004), 397-407.

16. Q.J. Zhang and K.C. Gupta, Neural networks for RF and microwave design, Artech House, Norwood, MA, 2000.

17. C. Yildiz and M. Turkmen, A CAD approach based on artificial neural networks for Shielded multilayered coplanar waveguide, AEUE Int J Electron and Commun 58 (2004), 1-9.

18. X. Ding, V. Devabhaktuni, B. Chattaraj, M. Yagoub, M. Deo, J. Xu, and Qi Jun Zhang, Neural-network approaches to electromagnetic-based modeling of passive components and their applications to high-frequency and high-speed nonlinear circuit optimization, IEEE Trans Microwave Theory Tech 52 (2004), 436449.

19. J. Rayas-Sanchez, EM-based optimization of micro- wave circuits using artificial neural networks: The stateof-the-art, IEEE Trans Microwave Theory Tech 52 (2004), 420-435.

20. R.S. Chen, X. Zhang, K.F. Tsang, and K.N. Yung, Modeling and design of interdigital capacitor based on neural networks and genetic algorithm, Microwave Opt Technol Lett 38 (2003), 231-235.

21. V. Devabhaktuni, C. Xi, F. Wang, and Qi-Jun Zhang, Robust training of microwave neural models, Int $\mathrm{J}$ Microwave and Millimeter-Wave CAE 12 (2002), 109-124.

22. J. Jargon, K.C. Gupta, and D. DeGroot, Applications of artificial neural networks to RF and microwave measurements, Int J Microwave and Millimeter-Wave CAE 12 (2002), 3-24.

23. S. Haykin, Neural networks: A comprehensive foundation, Macmillan College Publishing, New York, 1994.

24. J. Kennedy and R.C. Eberhart, Particle swarm optimization, Proc IEEE Conf Neural Networks IV (1995), 1942-1948.

25. R.C. Eberhart and Y. Shi, Particle swarm optimization: developments, applications and resources, IEEE Congress Evolutionary Computat, Seoul, Korea, 2001, pp. 81-86.

26. D.W. Boeringer and D.H. Werner, Particle swarm optimization versus genetic algorithms for phased array synthesis, IEEE Trans Antennas Propagat 52 (2004), 771-779.

27. G. Ciuprina, D. Ioan, and I. Munteanu, Use of intelligent-particle swarm optimization in electromagnetics, IEEE Trans Magn 38 (2004), 1037-1040.

28. J. Park, K. Choi, and D.J. Allstot, Parasitic-aware RF circuit design and optimization, IEEE Trans Circ Syst I 51 (2004), 1953-1966.

29. I.C. Trelea, The particle swarm optimization algorithm: convergence analysis and parameter selection, Info Processing Lett 85 (2003), 317-325.

30. K.E. Parsopoulos and M.N. Vrahatis, Recent approaches to global optimization problems through particle swarm optimization, Natural Computing 1 (2002), 235-306.

31. IE3D, Zeland Software, Inc., CA.

32. D. Pozar, Microwave engineering, $3^{\text {rd }}$ ed., 2005, Wiley, New York, pp. 187-188. 


\section{BIOGRAPHIES}

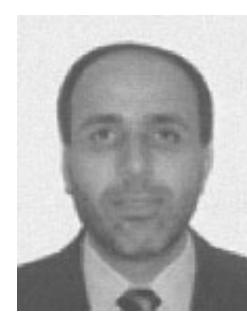

Jehad I. Ababneh received his B.S. degree from Jordan University of Science and Technology, Irbid, Jordan, in 1989, his M.E. degree from Tennessee State University, Nashville, TN, in 1998 and his Ph.D. degree from Wichita State University, Wichita, KS, in 2001, all in electrical engineering. He was a communication engineer with the Jordan Armed Forces, from 1990 to 1996. In 2001, he joined the American Tower Corporation, Georgia, as a Senior Engineer developing and delivering courses in wireless communication. He is currently with the Electrical Engineering Department of Jordan University of Science and Technology, Irbid, Jordan. His research interests include wireless communication, artificial intelligence, digital signal processing, and speech processing, modeling, and recognition.

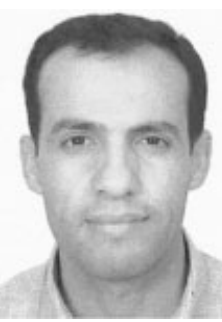

Majid M. Khodier received B.Sc. and M.Sc. degrees from Jordan University of Science and Technology, Irbid, Jordan, in 1995 and 1997, and his Ph.D. degree from The University of New Mexico, Albuquerque, in 2001, respectively, all in electrical engineering. He worked as a Postdoctorate in the department of electrical engineering at the University of New Mexico, where he performed research in the areas of RF/photonic antennas for wireless communications, and modeling of MEMS switches for multiband antenna applications. In September 2002, he joined the Department of Electrical Engineering at Jordan University of Science and Technology as an Assistant Professor. His research interests are in the areas of numerical techniques in electromagnetics, modeling of passive and active microwave components and circuits, applications of MEMS in antennas, and RF/Photonic antenna applications in broadband wireless communications. He has published more than 20 papers in journals and refereed conferences. He is a member of the IEEE Antennas and Propagation and Microwave Theory and Techniques Societies.

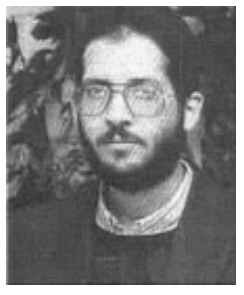

Nihad Dib received B.Sc. and M.Sc. degrees in electrical engineering from Kuwait University in 1985 and 1987, respectively. He obtained his Ph.D. in electrical engineering (majoring in electromagnetics and microwaves) in 1992 from University of Michigan, Ann Arbor. Then, he worked as an Assistant Research Scientist in the Radiation Laboratory at the same university. In September 1995, he joined the Electrical Engineering Department of Jordan University of Science and Technology (JUST) as an assistant professor, and became an Associate Professor in September 2000. His research interests are in computational electromagnetics and modeling of planar circuits. 